\title{
A CAUCHY CRITERION AND A CONVERGENCE THEOREM FOR RIEMANN-COMPLETE INTEGRAL
}

\author{
H. W. PU
}

(Received 10 October 1969; revised 8 January 1970)

Communicated by B. Mond

In 1957 Kurzweil [1] proved some theorems concerning a generalized type of differential equations by defining a Riemann-type integral, but he did not study its properties beyond the needs of that research. This was done by $R$. Henstock $[2,3]$, who named it a Riemann-complete integral. He showed that the Riemanncomplete integral includes the Lebesgue integral and that the Levi monotone convergence theorem holds. The purpose of the present paper is to give a necessary and sufficient condition for a function to be Riemann-complete integrable and to establish a termwise integration theorem for a uniformly convergent sequence of Riemann-complete integrable functions.

Throughout this paper, all functions considered are real-valued and defined in a closed interval $[a, b]$.

Definition 1. A division $\mathcal{D}$ of $[a, b]$ consists of two finite sequences $\left\{x_{j}\right\}_{j=0}^{n}$ and $\left\{z_{j}\right\}_{j=1}^{n}$ with conditions:

and

$$
a=x_{0}<x_{1}<\cdots<x_{n}=b
$$

$$
x_{j-1} \leqq z_{j} \leqq x_{j} \quad(j=1, \cdots, n) .
$$

Definition 2. A division $\mathfrak{D}$ of $[a, b]$ is said to be compatible with $\delta(z)>0$ defined in $[a, b]$ if, for each $j=1, \cdots, n,\left|x_{j}-z_{j}\right|<\delta\left(z_{j}\right)$ and $\left|z_{j}-x_{j-1}\right|<\delta\left(z_{j}\right)$.

It should be noted that there is at least a division $\mathscr{D}$ of $[a, b]$ which is compatible with a given function $\delta(z)>0$ defined in $[a, b)]$ [3, p. 83].

Definition 3. A function $f$ is Riemann-complete integrable in $[a, b]$ with integral $I(f)$ if there is a real number $I(f)$ such that to each $\varepsilon>0$ there corresponds a function $\delta(z)>0$ defined in $[a, b]$ with

$$
\left|\sum_{j=1}^{n} f\left(z_{j}\right)\left(x_{j}-x_{j-1}\right)-I(f)\right|<\varepsilon
$$

for all sums over divisions $\mathfrak{D}$ of $[a, b]$ compatible with $\delta(z)$.

In the sequel, we shall simply use the terms 'integrable' and 'integral' for 
'Riemann-complete integrable in $[a, b]$ ' and 'Riemann-complete integral in $[a, b]$ ' respectively. Also, for simplicity, we shall replace the words 'sum over a division of $[a, b]$ compatible with $\delta(z)$ ' by 'sum over $(\mathfrak{D}, \delta)$ '.

LEMMA 4. If $f$ and $g$ are integrable and $\alpha, \beta$ are real numbers, then $\alpha f+\beta g$ is also integrable and $I(\alpha f+\beta g)=\alpha I(f)+\beta I(g)$.

Proof. Let $\varepsilon>0$ be given. If $\alpha \neq 0$, since $f$ is integrable, there corresponds a function $\delta_{f}(z)>0$ defined in $[a, b]$ such that

$$
\left|S_{f}-I(f)\right|<\varepsilon /(2|x|)
$$

for all sums for $f$ over $\left(\mathscr{D}, \delta_{f}\right)$. Clearly we have, for any $\alpha$,

$$
\left|S_{\alpha f}-\alpha I(f)\right|=|\alpha|\left|S_{f}-I(f)\right|<\varepsilon / 2
$$

for all sums for $\alpha f$ over $\left(D, \delta_{f}\right)$. Similarly, there also corresponds a function $\delta_{g}(z)>0$ defined in $[a, b]$ such that

$$
\left|s_{\beta g}-\beta I(g)\right|<\varepsilon / 2
$$

for all sums for $\beta g$ over $\left(\mathscr{D}, \delta_{g}\right)$.

Let $\delta(z)=\min \left\{\delta_{f}(z), \delta_{g}(z)\right\}$ for all $z$ in $[a, b]$. Thus

$$
\left|S_{\alpha f+\beta g}-(\alpha I(f)+\beta I(g))\right| \leqq\left|S_{\alpha f}-\alpha I(f)\right|+\left|S_{\beta g}-\beta I(g)\right|<\varepsilon
$$

for all sums for $\alpha f+\beta g$ over $(\mathscr{D}, \delta)$. The proof is completed.

Definition 5. Let $\mathscr{D}$ be the set of all pairs $(\mathscr{D}, \delta)$, where $\delta$ is a positive function defined in $[a, b]$, and $D$ is a division of $[a, b]$ compatible with $\delta$. For each function $f$, define $S_{f}: \mathscr{D} \rightarrow R$ by setting $S_{f}(\mathscr{D}, \delta)$ to be the sum for $f$ over $(\mathfrak{D}, \delta)$.

Definition 6. Let $\left(\mathfrak{D}_{i}, \delta_{i}\right) \in \mathscr{D}, i=1,2$. We shall say $\left(\mathfrak{D}_{1}, \delta_{1}\right)<\left(\mathfrak{D}_{2}, \delta_{2}\right)$ if $\delta_{2} \leqq \delta_{1}$. Clearly, this is a partial ordering of $\mathscr{D}$.

Lemma 7. For any $f, S_{f}: \mathscr{D} \rightarrow R$ is a net.

Proof. We need only show that $\{\mathscr{L},<\}$ is a directed set. Let $\left(\mathscr{D}_{i}, \delta_{i}\right) \in \mathscr{D}$, $i=1,2$, be given. Define $\delta_{0}$ by $\delta_{0}(z)=\min \left\{\delta_{1}(z), \delta_{2}(z)\right\}$ for all $z \in[a, b]$. Then for any division $\mathscr{D}_{0}$ compatible with $\delta_{0},\left(\mathscr{D}_{0}, \delta_{0}\right) \in \mathscr{D}$ and $\left(\mathscr{D}_{i}, \delta_{i}\right)<\left(\mathscr{D}_{0}, \delta_{0}\right)$, $i=1,2$.

For a function $f$, we consider the following

Condition 8. To each $\varepsilon>0$ there corresponds a function $\delta(z)>0$ defined in $[a, b]$ with $\left|S^{\prime}-S^{\prime \prime}\right|<\varepsilon$ whenever $S^{\prime}$ and $S^{\prime \prime}$ are sums for $f$ over divisions compatible with $\delta(z)$.

Lemma 9. Condition 8 is necessary and sufficient for the net $S_{f}: \mathscr{D} \rightarrow R$ to be Cauchy.

Proof. Sufficiency: Let $\varepsilon>0$ be given. Consider the function $\delta(z)>0$ 
stated in condition 8 , it is immediate that $\left|S_{f}\left(\mathscr{I}_{1}, \delta_{1}\right)-S_{f}\left(\mathscr{D}_{2}, \delta_{2}\right)\right|<\varepsilon$ for any $\left(\mathscr{D}_{i}, \delta_{i}\right) \in \mathscr{D}$ such that $(\mathscr{D}, \delta)<\left(\mathscr{D}_{i}, \delta_{i}\right), i=1,2$, where $\mathscr{D}$ is an arbitrary division compatible with $\delta(z)>0$. The proof for necessity is similar.

Since $R$ is a complete uniform space, a Cauchy net $S_{f}: \mathscr{D} \rightarrow R$ has a limit in $R$ [4, pp. 193-194]. Thus we obtain the following Cauchy criterion:

THeOREM 10. A function $f$ is integrable if and only if it satisfies condition 8 , or equivalently, if and only if the net $S_{f}: \mathscr{D} \rightarrow R$ is Cauchy.

THEOREM 11. If $\left\{f_{n}\right\}$ is a sequence of integrable functions and converges uniformly to $f$ in $[a, b]$, then $f$ is integrable with integral $I(f)=\lim _{n \rightarrow \infty} I\left(f_{n}\right)$.

Proof. Let $\varepsilon>0$ be given, choose a positive number $\eta<\varepsilon /(4(b-a))$. By hypothesis, there is an $n_{0}$ such that

$$
\left|f_{n}(z)-f(z)\right|<\eta \text { for every } z \in[a, b] \text { and every } n \geqq n_{0} .
$$

Since $f_{n_{0}}$ is integrable, there exists $\left(\mathscr{D}^{*}, \delta^{*}\right) \in \mathscr{D}$ such that

$$
\left|S_{f_{n_{0}}}\left(\mathfrak{D}_{1}, \delta_{1}\right)-S_{f_{n_{0}}}\left(\mathfrak{D}_{2}, \delta_{2}\right)\right|<\varepsilon / 2 \quad \text { whenever }\left(\mathfrak{D}^{*}, \delta^{*}\right)<\left(\mathfrak{D}_{i}, \delta_{i}\right),
$$

$i=1,2$. Thus we have

$$
\begin{aligned}
\mid S_{f}\left(\mathfrak{D}_{1},\right. & \left.\delta_{1}\right)-S_{f}\left(\mathfrak{D}_{2}, \delta_{2}\right) \mid \\
& \leqq \\
\quad & \left|S_{f}\left(\mathfrak{D}_{1}, \delta_{1}\right)-S_{f_{n_{0}}}\left(\mathfrak{D}_{1}, \delta_{1}\right)\right|+\left|S_{f_{n_{0}}}\left(\mathfrak{D}_{1}, \delta_{1}\right)-S_{f_{n_{0}}}\left(\mathfrak{D}_{2}, \delta_{2}\right)\right| \\
& +\left|S_{f_{n_{0}}}\left(\mathfrak{D}_{2}, \delta_{2}\right)-S_{f}\left(\mathfrak{D}_{2}, \delta_{2}\right)\right|<\eta(b-a)+\varepsilon / 2+\eta(b-a)<\varepsilon \\
& \text { whenever }\left(\mathfrak{D}^{*}, \delta^{*}\right)<\left(\mathfrak{D}_{i}, \delta_{i}\right), \quad i=1,2 .
\end{aligned}
$$

It follows from theorem 10 that $f$ is integrable.

It remains to show that $\lim _{n \rightarrow \infty} I\left(f_{n}\right)=I(f)$. For this purpose, let $\varepsilon>0$ be given and $f_{n_{0}}$ be the same as above. Since $f$ and all $f_{n}$ are integrable, there exist $\left(\mathfrak{T}_{0}, \delta_{0}\right)$ and $\left(\mathfrak{D}_{n}, \delta_{n}\right)$ for each $n$ such that

and

$$
\left|S_{f}(\mathfrak{D}, \delta)-I(f)\right|<\varepsilon / 4 \text { whenever }\left(\mathfrak{I}_{0}, \delta_{0}\right)<(\mathfrak{D}, \delta)
$$

$$
\left|S_{f_{n}}(\mathfrak{D}, \delta)-I\left(f_{n}\right)\right|<\varepsilon / 4 \text { whenever }\left(\mathfrak{D}_{n}, \delta_{n}\right)<(\mathfrak{D}, \delta) \text {, for each } n \text {. }
$$

Evidently, for $n \geqq n_{0}$ and $(\mathscr{D}, \delta) \in \mathscr{D}$, we have

$$
\begin{aligned}
\left|I\left(f_{n}\right)-I(f)\right| & \left.\leqq\left|I\left(f_{n}\right)-S_{f_{n}}(\mathfrak{D}, \delta)\right|+\mid S_{f_{n}}(\mathfrak{D}, \delta)-S_{f}(\mathfrak{D}, \delta)\right\}+\left|S_{f}(\mathfrak{D}, \delta)-I(f)\right| \\
& <\varepsilon / 4+\left|S_{f}(\mathfrak{D}, \delta)-I(f)\right|+\left|S_{f_{n}}(\mathfrak{D}, \delta)-I\left(f_{n}\right)\right|
\end{aligned}
$$

and the last two terms can be made less than $\varepsilon / 2$ by choosing $(\mathscr{D}, \delta)$ in $\mathscr{D}$ with $\left(\mathfrak{I}_{0}, \delta_{0}\right)<(\mathfrak{D}, \delta)$ and $\left(\mathfrak{D}_{n}, \delta_{n}\right)<(\mathfrak{D}, \delta)$. The proof is completed.

COROLLARY 12. If $E \subset[a, b]$ is a Lebesgue null set and $\left\{f_{n}\right\}$ a sequence of integrable functions which converges to $f$ uniformly on $[a, b]-E$, then $f$ is integrable with integral $I(f)=\lim _{n \rightarrow \infty} I\left(f_{n}\right)$. 
Proof. For each $n$ let $g_{n}$ and $h_{n}$ be functions defined by

$$
\begin{aligned}
g_{n}(z) & =f_{n}(z) \text { for } z \in[a, b]-E, \\
& =0 \quad \text { for } z \in E,
\end{aligned}
$$

and

$$
h_{n}=f_{n}-g_{n} .
$$

Similarly, we define $g$ and $h$ by

$$
\begin{aligned}
g(z) & =f(z) \text { for } z \in[a, b]-E, \\
& =0 \quad \text { for } z \in E
\end{aligned}
$$

and

$$
h=f-g .
$$

It is trivial from the hypothesis that the sequence $\left\{g_{n}\right\}$ converges uniformly to $g$ on $[a, b]$ and that the functions $h$ and all $h_{n}$ are Lebesgue null. Since the integral considered here includes the Lebesgue integral, $h$ and all $h_{n}$ are integrable with $I(h)=I\left(h_{n}\right)=0$ for all $n$. Since for each $n g_{n}=f_{n}-h_{n}$ and by hypothesis $f_{n}$ is integrable, in view of lemma 4 , each $g_{n}$ is integrable and $I\left(g_{n}\right)=I\left(f_{n}\right)$. By theorem $11, g$ is integrable with $I(g)=\lim _{n \rightarrow \infty} I\left(g_{n}\right)$. By lemma 4 again, $f=h+g$ is integrable and $I(f)=I(g)=\lim _{n \rightarrow \infty} I\left(g_{n}\right)=\lim _{n \rightarrow \infty} I\left(f_{n}\right)$.

It is worth while noting that the above corollary is not true for Riemann integrals.

The author is deeply grateful to Dr. H. H. Pu for her valuable suggestion.

\section{References}

[1] J. Kurzweil, 'Generalized ordinary differential equations and continuous dependence on a parameter', Czechoslovak Math. Jour. 7 (82) (1957), 418-446.

[2] R. Henstock, Theory of Integration (Butterworths, London, 1963).

[3] R. Henstock, 'A Riemann-type integral of Lebesgue power', Canad. J. Math. 20 (1968), 79-87.

[4] J. L. Kelley, General Topology (Van Nostrand, Princeton, 1965).

Texas A\&M University

College Station, Texas

U.S.A. 